\title{
A New Cellular Architecture Based on an Interleaved Cluster Concept
}

\author{
Li-Chun Wang, Member, IEEE
}

\begin{abstract}
Personal communications services (PCS's) and future broad-band wireless systems are and will continue to be driven mainly by three challenging objectives: high-spectrum reuse efficiency, high-quality channels, and low infrastructure costs. We propose a new interleaved narrow-beam trisector cell (INBTC) architecture with a newly developed interleaved channel assignment (ICA) scheme to achieve these three objectives. Originating from the ICA scheme, we introduce the concept of interleaved cluster, within which a channel set can be assigned more than once. The INBTC architecture with interleaved clusters can enhance the performance of cellular/PCS systems from several perspectives. We demonstrate that to achieve highspectrum efficiency, specifically in the range of reuse factors $N=2-7$, the INBTC system improves 3-5 dB at the 90th and 95th percentiles of signal-to-interference ratio (SIR) compared to conventional cellular systems. For a typical cellular environment, with a shadow fading standard deviation $\sigma=8$ $\mathrm{dB}$ and an SIR requirement of $17 \mathrm{~dB}$ at the 90th percentile, the INBTC system can increase system capacity by $75 \%$ over current systems. Because the INBTC architecture can utilize the basestation equipment of current cellular/PCS systems, implementing it should be simple and carry no attendant infrastructure costs. Hence, the proposed INBTC system (the cell layout plus the channel assignment scheme) has significant potential value in future cellular/PCS systems, as well as in broad-band wireless applications.
\end{abstract}

\section{INTRODUCTION}

$\mathbf{I}$ N CELLULAR mobile and personal communications, three major objectives need to be met: high-spectrum reuse efficiency, high-quality performance, and low infrastructure costs. This paper documents a new cellular architecture with a new channel assignment scheme to achieve these three goals simultaneously.

Most conventional cellular systems employ three $100^{\circ}$ to $120^{\circ}$ directional antennas at each base station, as shown in Fig. 1. We call this kind of cell the wide-beam trisector cell (WBTC). A newer cellular architecture used in some second generation digital cellular systems, such as the global systems for mobile telecommunications (GSM's) and IS-136, is the narrow-beam trisector cell (NBTC). An NBTC (also called a clover-leaf cell [1]) is composed of three $60^{\circ}$ to $70^{\circ}$ directional antennas (see Fig. 2). The six-sectored cellular architecture, i.e., one with six $60^{\circ}$ directional antennas at a base, was also proposed at one time for cellular systems, but had unsuccessful

Manuscript received January 19, 1998; revised August 17, 1998.

The author is with the Wireless Communications Research Department, AT\&T Laboratories, Red Bank, NJ 07701 USA (e-mail: lichun@ research.att.com).

Publisher Item Identifier S 0018-9545(99)07392-2.

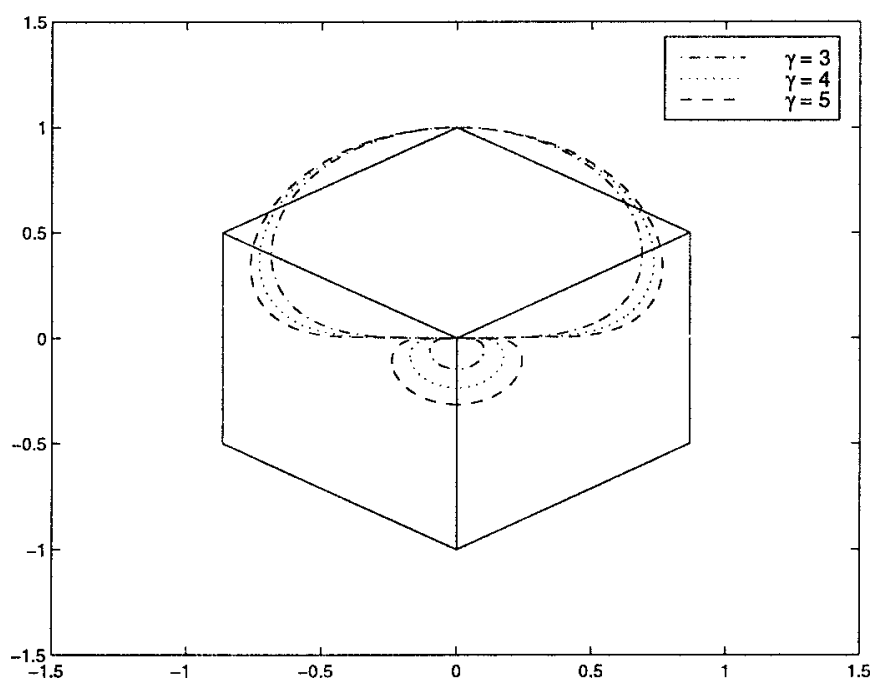

Fig. 1. Coverage area of a base station comprising three $120^{\circ}$ directional antennas, a wide-beam trisector cell (WBTC), where solid lines represent hypothetical sector contours, nonsolid lines analytical sector contours, and $\gamma$ is the path-loss exponent.

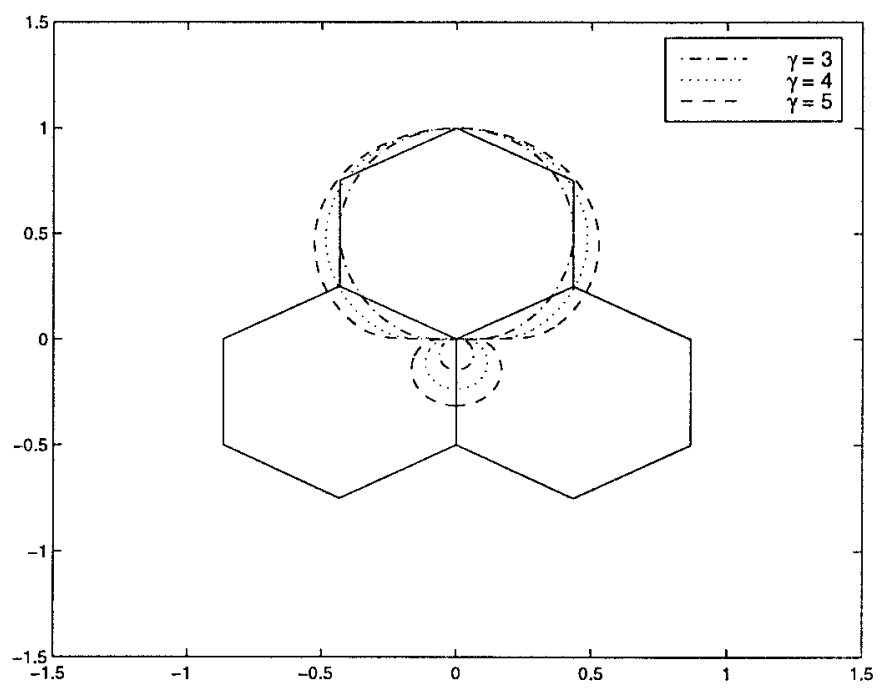

Fig. 2. Coverage area of a base station comprising three $60^{\circ}$ directional antennas, an NBTC, where solid lines represent hypothetical sector contours, nonsolid lines analytical sector contours, and $\gamma$ is the path-loss exponent.

field trials in the 1970's [2]. Although a six-sectored system has more degrees of freedom in managing bandwidth [3], it has lower trunking efficiency, requires more intracell handoffs, and has higher antenna costs compared with three-sectored systems. 
In this paper, we describe a new cellular architecture, called the interleaved NBTC (INBTC), to deliver the advantages of both the three-sectored and six-sectored systems, while avoiding their disadvantages. In [4], it is shown that the NBTC system outperforms the WBTC system, both in signal-to-interference ratio (SIR) performance and coverage performance. Because it utilizes the same narrow-beam sector concept, the INBTC system also has substantially better performance than the WBTC system. Furthermore, implementing the INBTC architecture will be simple and may carry no extra equipment costs because base-station equipment is already available in the current cellular/PCS systems using the NBTC architecture.

The difference between the proposed INBTC system and the NBTC system lies in the way the directional antenna base stations are placed. Specifically, in the NBTC system, base stations transmit signals in three main-beam directions throughout a whole service area, while in the INBTC system, we propose a sector rotation technique to obtain six main beam directions. This technique creates two types of NBTC's, each of which has three main-beam directions, as shown in Fig. 3. Putting two types of NBTC's in an interleaved pattern ensures complete coverage of the service area.

With six main-beam directions, the INBTC system has more flexibility in assigning channels than the NBTC system. To exploit this advantage fully, we have designed a new channel assignment scheme, called the interleaved channel assignment (ICA). This scheme assigns the same channel set to two different sectors with different beam directions in two adjacent cells so that they do not interfere with each other. Because the main-beam directions of cochannel sectors are pointing to two different angles alternately, we call the group of cells in the INBTC system that form a repeat pattern of the whole channel set the interleaved cluster. As an example in Fig. 4, cells $C_{1}$, $C_{2}, C_{3}$, and $C_{4}$ at the middle of the figure form an interleaved cluster. One important property for the interleaved cluster concept is that each channel set is reused more than once in the cluster and cochannel sets in the cluster do not cause interference to each other. Consequently, the INBTC system increases the frequency reuse efficiency, without sacrificing the SIR performance. By contrast, in a traditional cellular system, each channel set is assigned only once in a cluster, e.g., the shaded cells in Figs. 5 and 6, thereby resulting in lower reuse efficiency than in the INBTC system.

This paper is organized as follows. Section II further describes the INBTC system architecture. Section III presents the ICA algorithm and defines a generalized reuse factor that determines the spectrum efficiency of the INBTC system. To demonstrate the performance advantages of the INBTC system over the NBTC and WBTC systems, we first provide a worst case SIR analysis for the three systems in Section IV. Through a simulation study, we obtain more numerical results in Section V. Finally, Section VI provides some concluding remarks.

\section{System ARCHITECTURE}

In this section, we compare the INBTC architecture with conventional cellular architectures. The merits of the INBTC architecture are based on three key factors: 1) the narrow-beam antenna architecture (as compared to the WBTC architecture); 2) the sector rotation technique; and 3) the new site layout design.

\section{A. Antenna Architecture: NBTC Versus WBTC}

The INBTC system is designed to exploit the benefits of the antenna architecture used in the NBTC system. First, we briefly explain why the NBTC system is a better three-sectored system than the traditional WBTC system.

The traditional WBTC architecture uses three $100^{\circ}-120^{\circ}$ antennas at each cell site. A WBTC forms a coverage area with the shape of a hexagon, as shown in Fig 1. Note that coverage contours shown as the dashed lines are normalized to the signal strength at the azimuth angle of $\theta=0$ degree. We observe that the ideal diamond-shaped sector of a WBTC does not match the actual coverage contour of the antenna. Side-lobe levels are also significant in adjacent sectors. Furthermore, poor coverage occurs in the corners of the hexagon at the common boundary of two sectors.

In contrast, an NBTC is defined as the coverage area of a base station with three directional antennas, each of which has $60^{\circ}$ beamwidth. The coverage area of such an antenna is shown in Fig. 2, where the sector boundary is defined as the equal-signal-strength contour from the antenna. Note that the coverage area of a $60^{\circ}$ antenna can be closely approximated by a hexagon, and with three such antennas, the coverage contour of an NBTC is therefore like a clover leaf, as shown in Fig. 2. Because of the better match between the hypothetical cellular contour and the actual cellular contour, the performance of the NBTC system exceeds that of the WBTC system. A detailed performance comparison is reported in [4].

\section{B. Sector Rotation Technique}

Using the NBTC architecture, we propose a sector rotation technique to allow flexible and efficient channel assignment schemes. The concept of sector rotation was first introduced for the WBTC system [5] to create a low-interference region for installing underlaid microcells in a hierarchical cellular system. That technique does not increase the number of main-beam directions, as we do in this study.

The sector rotation technique in this study results into two types of cells. As shown in Fig. 3, the type-I cell has three sectors labeled 1,2 , and 3 pointing in the directions of $30^{\circ}$, $150^{\circ}$, and $270^{\circ}$, respectively. For the type-II cell, these sectors are rotated by $60^{\circ}$ relative to the first type. In the following, we show how these two types of cells be tessellated for complete coverage of the entire service area.

\section{Site Layouts}

Site layouts can be obtained by tessellating the service area with the coverage area of each cellular architecture. Examples of site layouts for the INBTC, NBTC, and WBTC systems are shown in Figs. 4-6, respectively, where the NBTC and WBTC systems have a reuse factor $N=3$, and the INBTC system has a reuse factor $N=2$. To maintain the same separation between two adjacent cell sites for the three systems, we have 

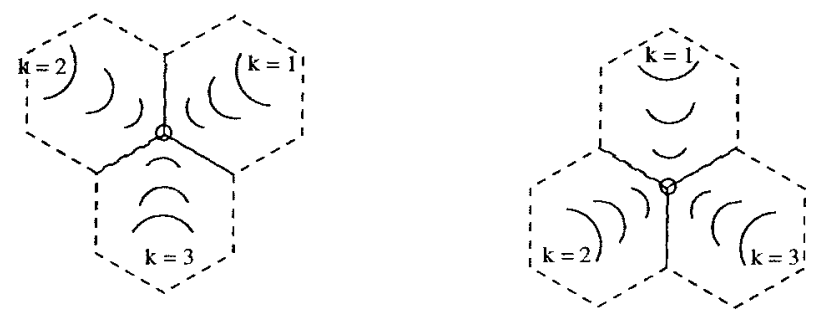

NBTC (Type -I)

NBTC (Type - II)
Fig. 3. Cell structures adopted in the proposed system, composing two types of NBTC's: one has $30^{\circ}-150^{\circ}-270^{\circ}$ orientation, and the other has $90^{\circ}-210^{\circ}-330^{\circ}$ orientation.

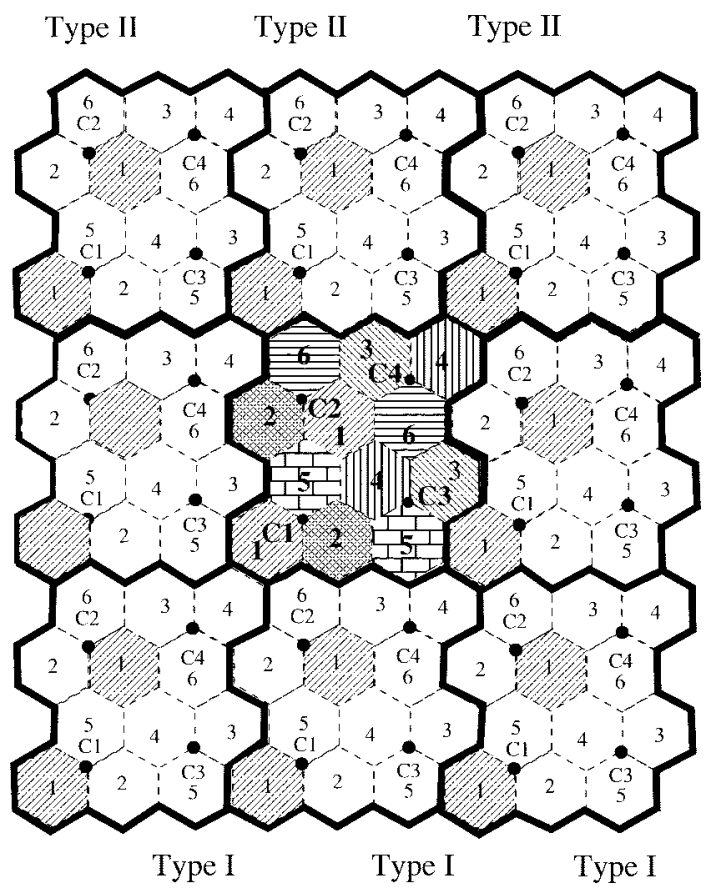

Fig. 4. The INBTC system with a reuse factor $N=2$.

the following relationship among the cell radii of the three architectures:

$$
R_{1}=R_{2}=\frac{2}{\sqrt{3}} R_{3}
$$

where $R_{1}, R_{2}$, and $R_{3}$ are the cell radius of the INBTC, NBTC, and WBTC, respectively. Because of this radius difference, to maintain the same received signal strength at the farthest points of an NBTC and a WBTC with the same transmitting power, the base station in an NBTC system needs about 2.5-dB higher antenna gain than that in the WBTC system, assuming a path-loss exponent $\gamma=4$. This requirement is easily met because $60^{\circ}$ antennas usually have $2-3-\mathrm{dB}$ higher gains than antennas with $100^{\circ}$ to $120^{\circ}$ beamwidth. The relation in (1) also implies that the area of each sector is equal for the three systems. Thus, even with the "interlocking" nature between cells, the INBTC system can still provide as complete coverage as the NBTC and WBTC systems, without increasing cell sites.

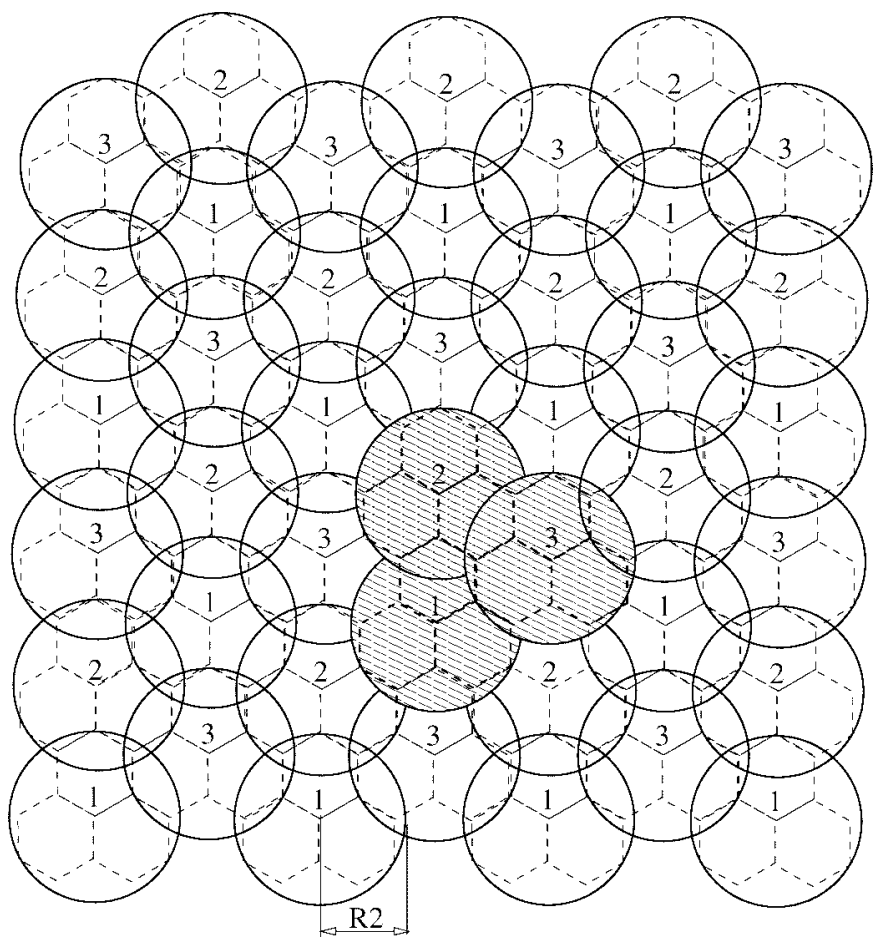

Fig. 5. Site layout for the NBTC system with a reuse factor $N=3$.

For the INBTC system, as shown in Fig. 4, cells in the vertical direction are of the same type, while two different cell types interleave in the horizontal direction. This is also one of the reasons for naming this system the interleaved NBTC system. Another reason is the characteristics of the ICA scheme proposed for the INBTC system, as discussed in more detail in the next section.

\section{InTERleAVEd ChANNEl AsSignMent AND GENERALIZED REUSE FACTOR}

\section{A. Generalized Reuse Factor}

According to the classical definition, a cluster is defined as a group of cells to which all channels available to the system are assigned once. Thus, the channel repeat pattern is determined by the cluster, and the cluster size becomes the reuse factor. In Section I, we introduce the concept of the interleaved cluster as the channel repeat pattern, within which a channel set can be assigned to more than one sector. Consequently, we define the generalized reuse factor $N$ as following:

$$
N=\frac{x}{y \times z}
$$

where $x$ is the number of sectors in the channel repeat pattern, $y$ is the number of sectors per cell, and $z$ is the number of times the same channel set is used in the pattern. This definition is more general in the sense that conventional cellular systems restrict $z=1$, whereas the INBTC system allows $z \geq 1$. The definition in (2) implies that channel assignment schemes directly determine the channel repeat pattern, the cluster size, and, therefore, the reuse factor. 


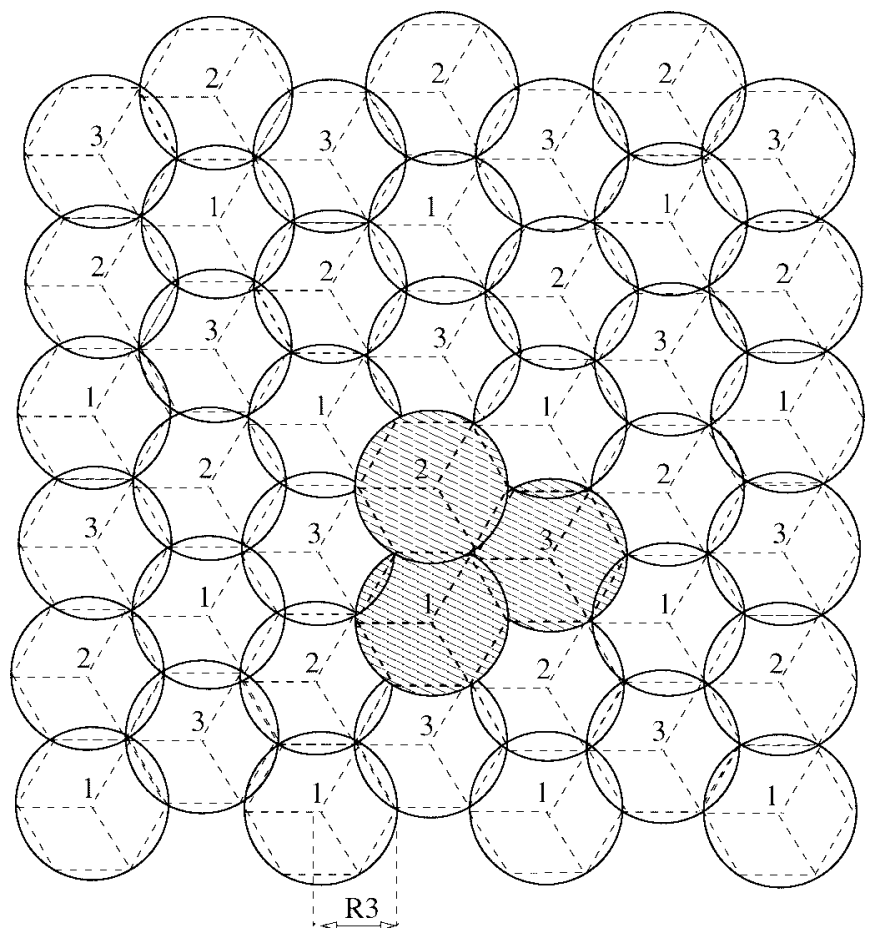

Fig. 6. Site layout for the WBTC system with a reuse factor $N=3$.

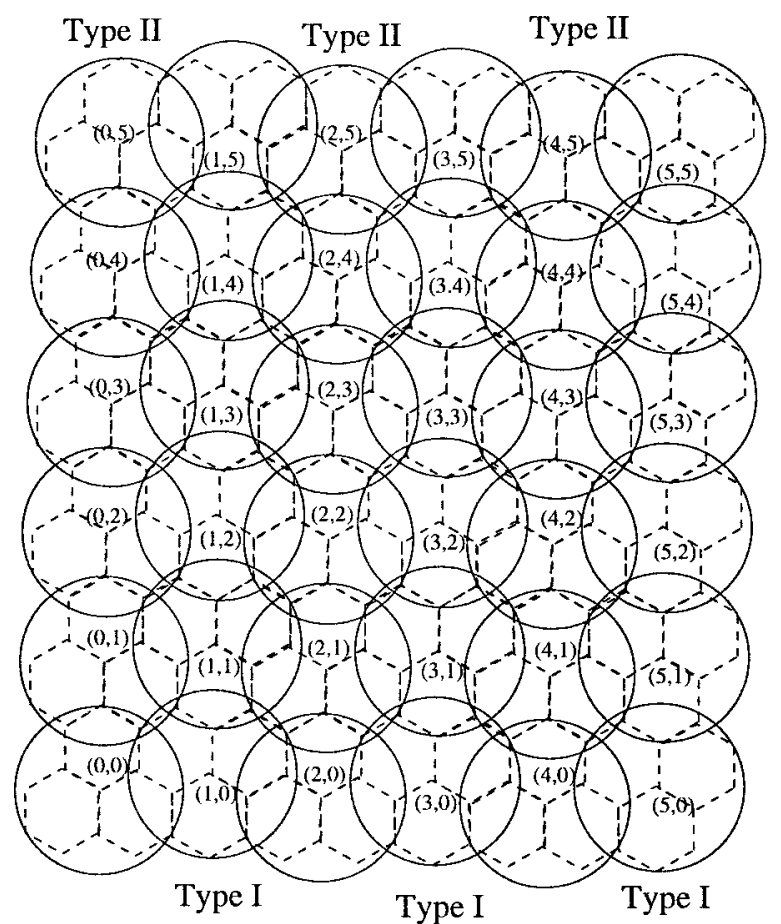

Fig. 7. Cell indexes for the INBTC system.

\section{B. Interleaved Channel Assignment}

We start with a simple case, $N=2$. Consider the system depicted in Fig. 4, which utilizes a cluster of four cells, labeled C1, C2, C3, and C4, each of which has three sectors. The total spectrum available to the system is partitioned into six channel sets. Each sector is assigned with a single channel set from 1 to 6 , with each channel set being reused a second time by a

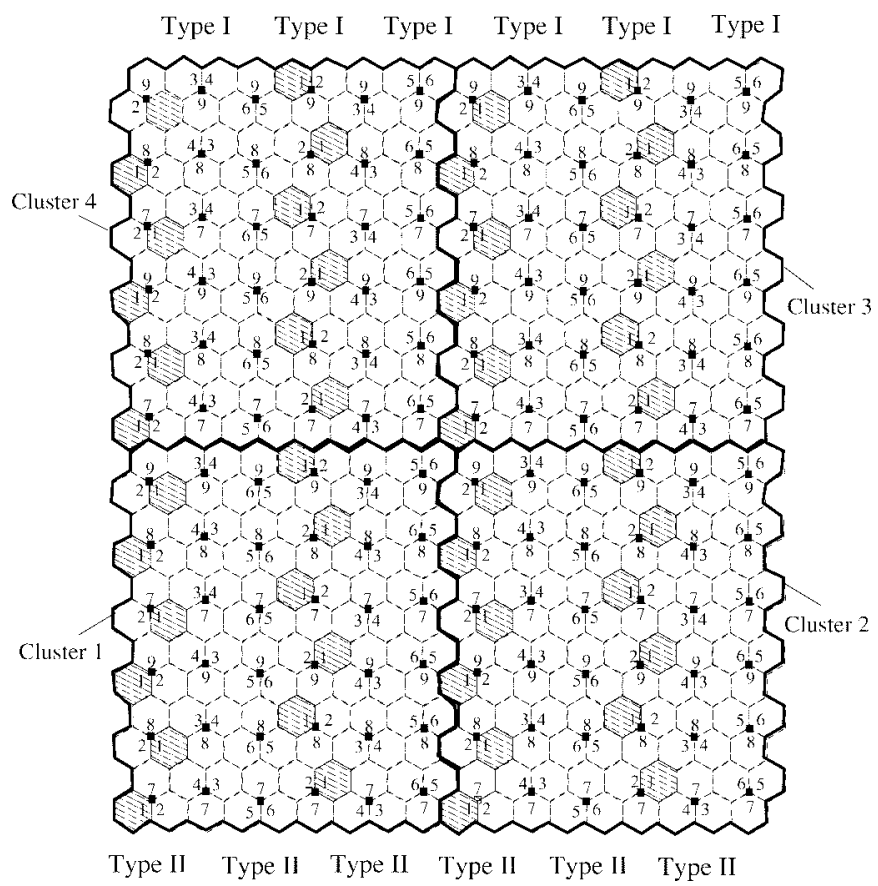

Fig. 8. The INBTC system with a reuse factor $N=3$.

sector in an adjacent cell. As shown in Fig. 4, channel sets 1,2 , and 5 are assigned for cell $\mathrm{C} 1$; sets 1,2 , and 6 for cell C2; sets 3, 4, and 5 for cell C3; and sets 3, 4, and 6 for cell $\mathrm{C} 4$. The resulting pattern yields at least one sector separation between cochannel sectors. Furthermore, the cochannel sectors in a cluster are pointing in different directions so that these sectors will not interfere with each other, thereby minimizing cochannel interference. This pattern is repeated throughout the service area. It also ensures that each channel set is used twice for every cluster of four cells (or 12 sectors). From the definition (2), we have $x=12, y=3$, and $z=2$, thereby obtaining a reuse factor $N=2$.

To generalize the above channel assignment for any given integer reuse factors $N \geq 2$, we first establish the following notations.

- Consider a rectangular coverage area served by an INBTC system with $L \times M$ base stations (cells) denoted by $C(i, j)$ for $i=0, \cdots L-1$ and $j=0, \cdots, M-1$. An example with $L=M=6$ is shown in Fig. 7 .

- Assume that types I and II cells are employed at base stations $C(i, j)$ in odd- and even-numbered columns (i.e., odd $i$ or even $i$ ), respectively.

- Let $S(i, j, k)$ denote the $k$ th sector of cell $C(i, j)$ (see Fig. 3.).

With the above notation, the ICA scheme is described as follows.

\section{Interleaved Channel Assignment Scheme:}

1) For a given reuse factor $N \geq 2$, divide the whole available frequency spectrum into $3 N$ channel sets, denoted by $1,2, \cdots, 3 N$.

2) a) Start from the left-most column of cells $C(i, j)$ (i.e., $i=0)$.

b) Select channel set $\alpha \in\{1,3, \ldots, 2 N-1\}$. 


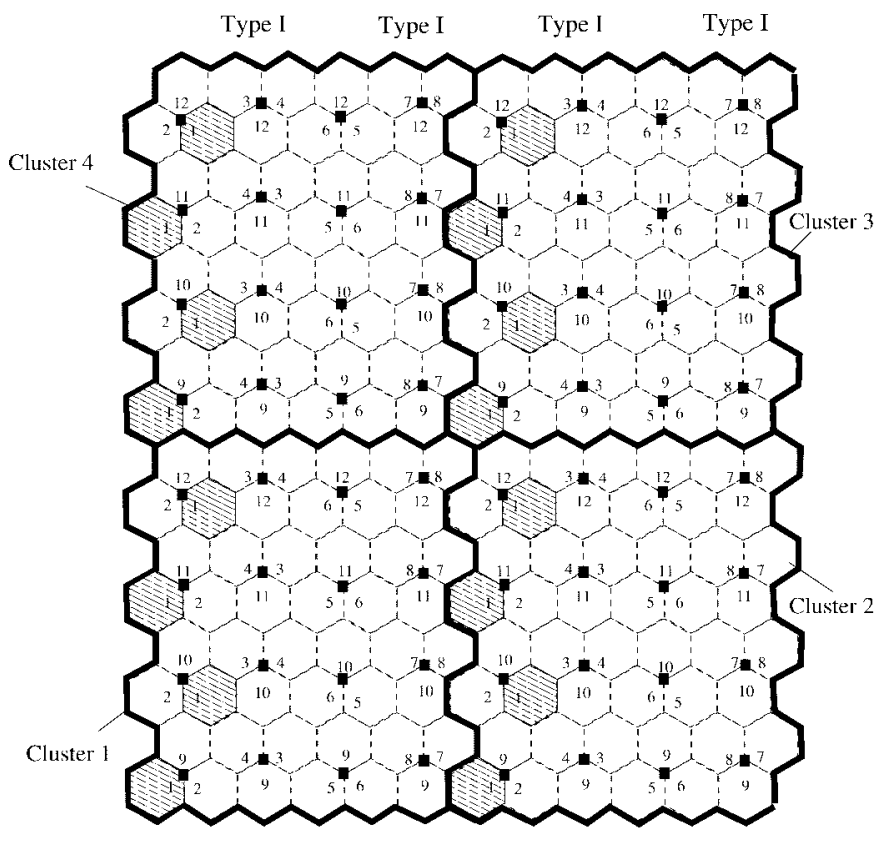

Type II Type II Type II Type II

Fig. 9. The INBTC system with a reuse factor $N=4$.

c) For $j=0$ to $M-1$, assign channel set $\alpha$ to sector $S([i / 2], j, k)$ and sector $S([i / 2], j, k+1)$ with $k=1$ if $C(i, j)$ is a type I cell and $k=2$, otherwise, where $[Y]$ is the largest integer less than $Y$.

d) Move to the next column of cells $(i=i+1)$.

e) Select a new channel set $\alpha=\bmod (\alpha+2,2 N-1)+1$.

f) Assign channel set $\alpha$ based on the rule described in Step $2(\mathrm{~b})$, where $\bmod (a, b)$ is the modular function with modulus $b$.

g) Repeat Steps 2(d)-(f) until the last column of base stations $S(L-1, j, k), j=0, \cdots, L-1$, is assigned with a channel set.

3 ) a) Start from the left-most column of cells $C(i, j)$ (i.e., $i=0$ ).

b) Select channel set $\beta \in\{2,4, \cdots, 2 N\}$.

c) For $j=0$ to $M-1$, assign channel set $\beta$ to sector $S([i / 2]-1, j, k)$ and sector $S([i / 2]-1, j, k+1)$ with $k=1$ if $C(i, j)$ is a type I cell and $k=2$, otherwise, where $[Y]$ is the largest integer less than $Y$.

d) Move to the next column of cells $(i=i+1)$.

e) Select a new channel set $\beta=\bmod (\beta+2,2 N)+1$.

f) Assign channel set $\beta$ based on the rule described in Step 3(c).

g) Repeat Steps 3(d)-(f) until the last column of base stations $S(L-1, j, k), j=0, \cdots, L-1$, is assigned with a channel set.

4) a) Start from the lowest row of cells $C(i, j)$ (i.e., $j=0)$.

b) Select channel set $\eta \in\{2 N+1, \cdots, 3 N\}$.

c) For $i=0$ to $L-1$, assign channel set $\eta$ to sector $S(i, j, 1)$ and sector $S(i, j, 3)$.

d) Move to the next row of cells $(j=j+1)$.

e) Select a new channel set $\eta=\bmod (\eta+1, N)+$ $2 N+1$. f) Assign channel set $\eta$ based on the rule described in Step 4(c).

g) Repeat Steps 4(d)-(f) until the last row of base stations $S(M-1, j, k), j=0, \cdots, L-1$, is assigned with a channel set.

Applying the above algorithm, we obtain channel sets assigned to the INBTC systems for the cases $N=3$ and $N=4$, as shown in Figs. 8 and 9, respectively. For $N=3$, observe from Fig. 8 that a channel repeat pattern is formed in a cluster of 36 cells (or equivalently, 108 sectors) located on an approximately square grid. Within these 108 sectors, each of nine channel sets is reused 12 times, thereby yielding the reuse factor $N=3$ from (2). For another example, Fig. 9 presents a pattern in a cluster of 16 cells (48 sectors), in which each of 16 channels sets is reused four times, thus yielding $N=4$.

To summarize, an INBTC system with the ICA scheme requires $3 N$ channel sets for a given reuse factor $N$. If $N$ is an odd integer, the repeat pattern of the whole channel sets is formed in a cluster of $4 N^{2}$ cells, within which the total channel sets available to the system are reused $4 N$ times. On the other hand, if $N$ is an even integer, a cluster of $N^{2}$ cells form a repeat pattern of the whole channel set, within which the total channel set is used $N$ times. The ICA scheme ensures that any two cochannel sectors are separated by at least a sector and the main beams of the nearest two cochannel sectors are pointing in two different directions so that they will not interfere with each other. Thus, the INBTC system simultaneously achieves high reuse efficiency and low interference. The detailed interference analysis for the INBTC system is discussed in the next section.

\section{PERFormance ANALYSIS}

In this section, we first describe the performance criteria, and then compare the performances of the WBTC, NBTC, and INBTC systems based on a worst case analysis.

\section{A. Performance Criteria}

The coverage probability, or reliability function, is defined as

$$
P\left(\lambda_{\mathrm{th}}\right) \triangleq \operatorname{Prob}\left[S / I>\lambda_{\mathrm{th}}\right]
$$

where $S$ and $I$ are the local mean of the desired received power and the total interference power, respectively. We have

$$
I=\sum_{i=1}^{n} I_{i}
$$

where $I_{i}$ is the received power from the $i$ th individual interferer and $n$ is the number of active cochannel interferers. ${ }^{1}$ For a given air interface, if the required (or threshold) value of SIR for good reception is $\lambda_{\mathrm{th}}$, then $P\left(\lambda_{\mathrm{th}}\right)$ is the coverage probability, or reliability, for that interface, and $\left[1-P\left(\lambda_{\mathrm{th}}\right)\right]$ is the outage probability.

\footnotetext{
${ }^{1}$ For studying SIR performance, we ignore the effects of receiver thermal noise.
} 


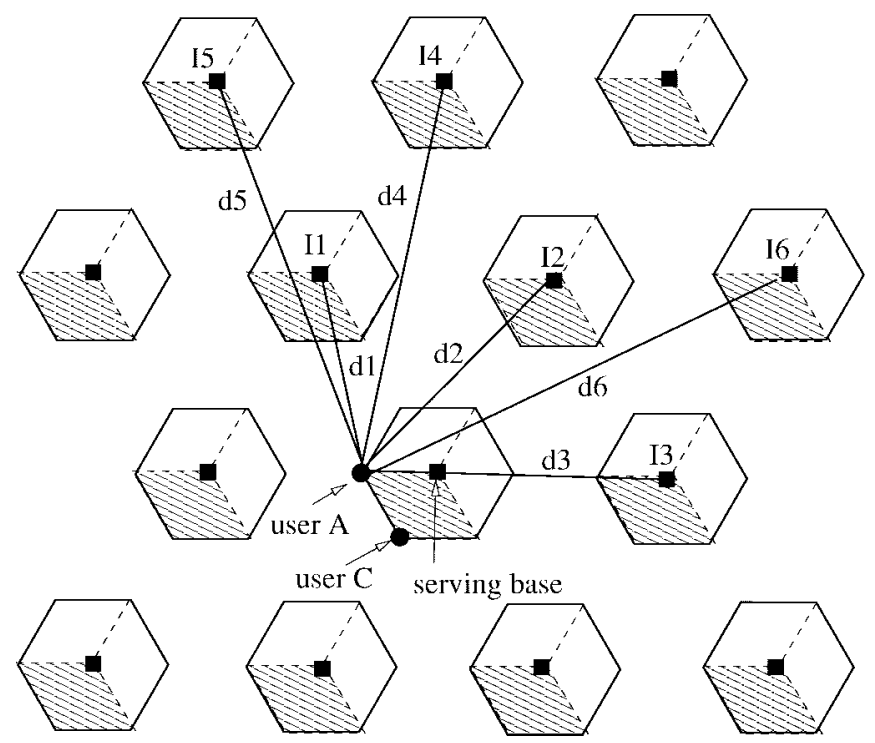

Fig. 10. The worst interference scenario for the WBTC system illustrated in Fig. $6(N=3)$.

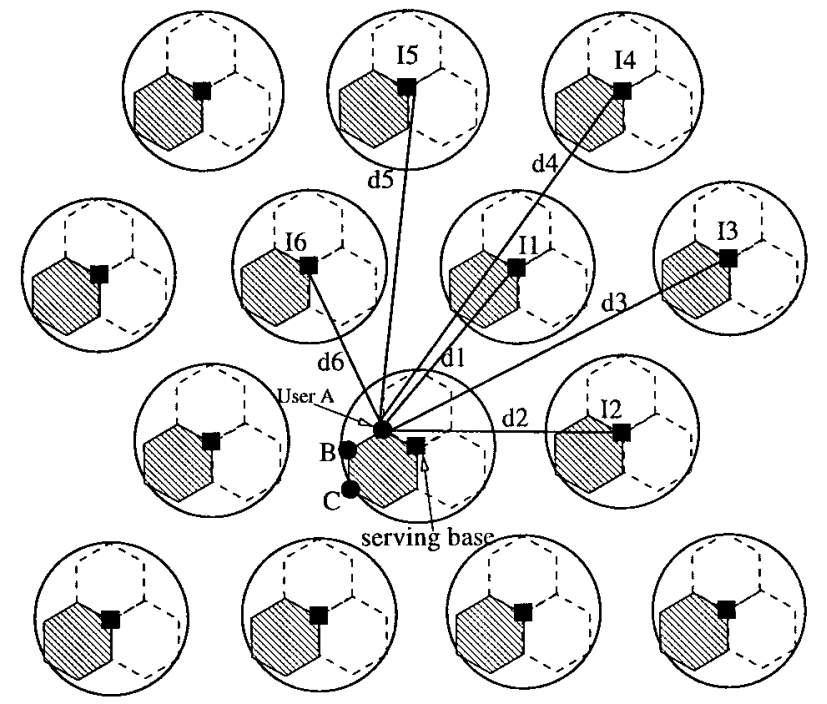

Fig. 11. The worst interference scenario for the NBTC system illustrated in Fig. $5(N=3)$.

Assuming that all sectors transmit at the same power, the SIR for a given user can be expressed as

$$
\frac{S}{I}=\left[\sum_{i=1}^{n}\left(\frac{G_{i}\left(\theta_{i}\right)}{G\left(\theta_{0}\right)}\right)\left(\frac{d_{i}}{d_{0}}\right)^{-\gamma}\left(\frac{\omega_{i}}{\omega_{0}}\right)\right]^{-1}
$$

where $\theta_{i}$ is the angles to the main beam of a directional antenna, $G\left(\theta_{i}\right)$ is the antenna gain at angle $\theta_{i}$ in decibels, $d_{i}$ is the distance of the $i$ th interferer to the user, $\omega$ is the shadow fading variable $[10 \log (\omega)$ is a Gaussian random variable with zero mean and standard deviation $\sigma$ ], the subscript 0 corresponds to the user (desired signal), and $i=1, \cdots, n$ are the indexes for the active cochannel interferers. We include the impact of side-lobe and back-lobe interference in this study by assuming realistic radiation patterns of directional antennas, from which Figs. 1 and 2 are produced.

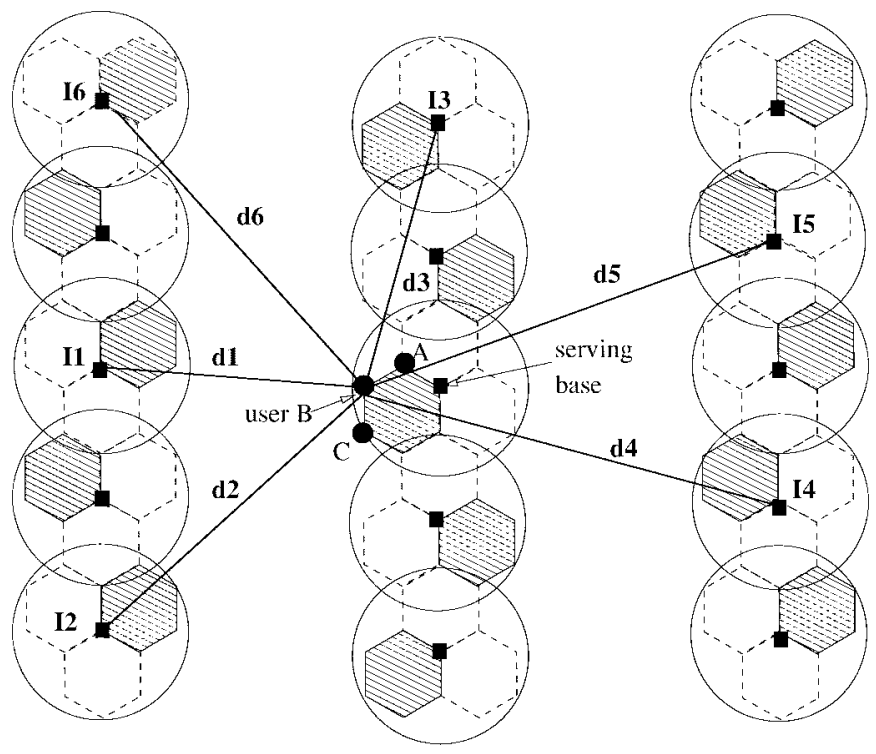

Fig. 12. The worst interference scenario for the INBTC system illustrated in Fig. $8(N=3)$.

TABLE I

INTERFERENCE ANALYSIS FOR THE WBTC SyStem $(N=3)$

\begin{tabular}{c|c|c|c|c}
\hline & & & & \\
& $d_{i} / R$ & $\theta_{i}(\mathrm{deg})$ & $G\left(\theta_{i}\right)(\mathrm{dB})$ & $\left(d_{i} / R\right)^{-\gamma} \times 10^{\frac{G\left(\theta_{i}\right)}{10}}$ \\
\hline$I_{1}$ & 2.65 & 40 & -1.16 & $1.56 \times 10^{-2}$ \\
$I_{2}$ & 3.61 & 13 & -0.11 & $5.77 \times 10^{-3}$ \\
$I_{3}$ & 4 & 60 & -2.88 & $2.01 \times 10^{-3}$ \\
$I_{4}$ & 5.29 & 19 & -0.24 & $1.21 \times 10^{-3}$ \\
$I_{5}$ & 5.57 & 51 & -2.01 & $6.55 \times 10^{-4}$ \\
$I_{6}$ & 6.08 & 34 & -0.814 & $6.06 \times 10^{-4}$ \\
\hline$S$ & 1 & 60 & -2.88 & 0.52 \\
\hline
\end{tabular}

\section{B. Worst Case S/I Analysis}

Neglecting the shadowing effect, the worst case cochannel interference scenarios for the WBTC, NBTC, and INBTC systems with $N=3$ are shown in Figs. 10-12, respectively, where a user is at the cell boundary of a serving base station. The locations of interfering base stations in the figures are obtained from Figs. 6 to 8. Assume the dominant sources of interference come from the strongest six cochannel interfering cells, whose distances to a user at cell boundary are represented as the six solid lines in Figs. 10-12. Because the received signal powers from the desired base station and the interfering based station vary with user locations, we examine the performance at two extreme user locations for the WBTC system, and three for the NBTC and INBTC systems. As shown in the figures, users $A$ and $B$ are positioned at the cell boundary and the angles from their locations to the main beam of a sectored antenna are $60^{\circ}$ and $30^{\circ}$, respectively. In addition, user $C$ is located at the farthest point of a cell in the main beam direction of a sectored antenna.

Compared to users in other cell boundary locations, we find that user $A$ is at the worst position in the WBTC and NBTC systems, and user $B$ is at the worst position in the INBTC system. For users $A$ in the WBTC and NBTC systems, and user $B$ in the INBTC system, Tables I-III, respectively, list 
TABLE II

INTERFERENCE ANALYSIS FOR THE NBTC System $(N=3)$

\begin{tabular}{c|c|c|c|c}
\hline & & & & \\
& $d_{i} / R$ & $\theta_{i}(\mathrm{deg})$ & $G\left(\theta_{i}\right)(\mathrm{dB})$ & $\left(d_{i} / R\right)^{-\gamma} \times 10^{\frac{G\left(\theta_{i}\right)}{10}}$ \\
\hline$I_{1}$ & 2.65 & 19 & -1.15 & $1.56 \times 10^{-2}$ \\
$I_{2}$ & 3.04 & 34 & -3.72 & $4.97 \times 10^{-3}$ \\
$I_{3}$ & 4.77 & 5 & -0.08 & $1.90 \times 10^{-3}$ \\
$I_{4}$ & 5.22 & 24 & -1.85 & $8.81 \times 10^{-4}$ \\
$I_{5}$ & 4.27 & 54 & -9.49 & $3.37 \times 10^{-4}$ \\
$I_{6}$ & 2.18 & 83 & -23.23 & $2.11 \times 10^{-4}$ \\
\hline$S$ & 0.5 & 60 & -11.37 & 1.17 \\
\hline
\end{tabular}

TABLE III

INTERFERENCE ANALYSIS FOR THE INBTC System $(N=3)$

\begin{tabular}{c|c|c|c|c}
\hline & & & & \\
& $d_{i} / R$ & $\theta_{i}(\mathrm{deg})$ & $G\left(\theta_{i}\right)(\mathrm{dB})$ & $\left(d_{i} / R\right)^{-\gamma} \times 10^{\frac{G\left(\theta_{i}\right)}{10}}$ \\
\hline$I_{1}$ & 3.04 & 34 & -3.72 & $4.97 \times 10^{-3}$ \\
$I_{2}$ & 4.09 & 12 & -0.46 & $3.21 \times 10^{-3}$ \\
$I_{3}$ & 3.12 & 43 & -5.97 & $2.66 \times 10^{-3}$ \\
$I_{4}$ & 4.92 & 15 & -0.72 & $1.44 \times 10^{-4}$ \\
$I_{5}$ & 5.07 & 50 & -8.12 & $2.33 \times 10^{-4}$ \\
$I_{6}$ & 4.44 & 76 & -19.02 & $3.21 \times 10^{-5}$ \\
\hline$S$ & 0.87 & 30 & -2.70 & 0.96 \\
\hline
\end{tabular}

TABLE IV

SIR in Different Locations for the Three Systems $(N=3)$

\begin{tabular}{c|c|c|c}
\hline Location & WBTC & NBTC & INBTC \\
\hline $\mathrm{A}$ & 12.93 & 16.88 & 19.42 \\
$\mathrm{~B}$ & - & 17.85 & 18.82 \\
$\mathrm{C}$ & 18.1 & 19.14 & 19.8 \\
\hline
\end{tabular}

the values of $d_{i} / R, \theta_{i}, G\left(\theta_{i}\right)$, and $\left(d_{i} / R\right)^{-\gamma} \times 10^{\frac{G\left(\theta_{i}\right)}{10}}$, where $d_{i} / R$ is the normalized distances of the interferers to the cell radius $R$ and $\left(d_{i} / R\right)^{-\gamma} \times 10^{\frac{G\left(\theta_{j}\right)}{10}}$ is the path gain. Substituting these values from Tables I-III into (5) with $\omega_{i}=1$ (i.e., no shadowing), we find the values of the worst case SIR are $12.93,16.88$, and $18.82 \mathrm{~dB}$ for the WBTC, NBTC, and INBTC systems, respectively. For purposes of comparison, Table IV lists the values of SIR for users $A, B$, and $C$ for these three systems. It is clear from this analysis that for a reuse factor $N=3$ and no shadowing, the NBTC system improves by about $4 \mathrm{~dB}$ in worst case SIR over the WBTC system, and the INBTC system further improves by about $2 \mathrm{~dB}$ in worst case SIR over the NBTC system.

The performance improvement of the NBTC system over the WBTC system is mainly due to the fact that the radiation pattern of a $60^{\circ}$ directional antenna closely matches a hexagonal sector in an NBTC, whereas the radiation pattern of a $120^{\circ}$ directional antenna poorly covers a diamond-shaped sector in a WBTC. The improvement of the INBTC system over the NBTC system, on the other hand, is attributed to the ICA algorithm, which reduces the interference power by arranging the antenna directions of the interfering sources. Specifically, the closer interfering base stations are assigned smaller antenna gains to reduce their impacts, while the farther base stations are given greater antenna gains. From Table III, for example, the nearest two interferers $I_{1}$ and $I_{3}$ for the INBTC system, which have distances about three times the cell radius, have the

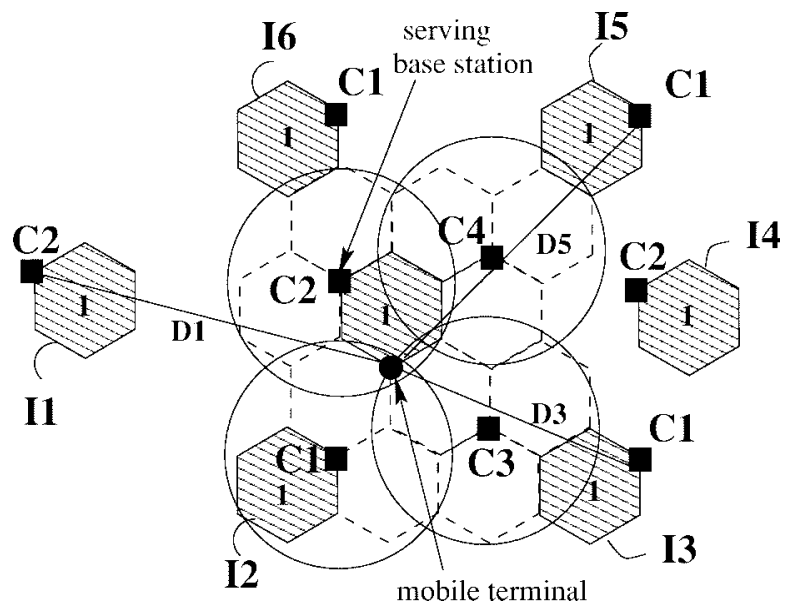

Fig. 13. An interference scenario for the INBTC system illustrated in Fig. 4 $(N=2)$.

normalized antenna gains -3.72 and $-5.97 \mathrm{~dB}$, respectively, while the farther interferers $I_{2}$ and $I_{4}$, are assigned larger antenna gains, -0.46 and $-0.72 \mathrm{~dB}$, respectively. On the other hand, from Tables I and II, we find that, for the strongest four interferers, $I_{1}$ to $I_{4}$, in the WBTC and NBTC systems, the shorter the normalized distance $d_{i} / R$ of interferers, the larger the impact on the user. As a result, the strongest interferer in the INBTC system has less impact than that in the WBTC and NBTC systems. From Tables I-III, we also find the path gain of $I_{1}$ in the INBTC system is $4.97 \times 10^{-3}$, but the path gains of $I_{1}$ in the WBTC and NBTC systems are $1.56 \times 10^{-2}$. In our example, among the second strongest interferers $I_{2}$ for the three systems, the interferer $I_{2}$ for the INBTC system has the least power. Since both the strongest two interferers $I_{1}$ and $I_{2}$ in the INBTC system have less impact than those in the WBTC and NBTC systems, the SIR performance of the INBTC system exceeds that of the WBTC and NBTC systems.

Another advantage of the INBTC system occurs when it is necessary to implement a very low reuse factor, such as $N=2$. In this case, the INBTC architecture can maintain a smaller SIR performance degradation compared to the WBTC and NBTC systems. In the NBTC and WBTC systems, the lowest reuse factor is $N=3$ to ensure at least a separation of one cell's distance to a major cochannel interfering base station. By implementing a reuse factor $N=2$ in the NBTC and WBTC systems, we inevitably create a major cochannel interfering base station adjacent to the desired cell, as shown in Fig. 14. One can expect that this scenario degrades the performance seriously. Even if site diversity is implemented, a user always encounters an interferer in an adjacent cell, thereby decreasing the site diversity gain.

Instead, even with a reuse factor $N=2$, two adjacent cochannel cells in the INBTC system do not interfere with each other because these two cochannel sectors are pointing in two different directions, as shown in Fig. 4. Specifically, consider a serving base station surrounded by six cochannel interfering base stations, $I_{1}$ to $I_{6}$, as shown in Fig. 13. Due to the ICA scheme, the nearest two interferers, $I_{2}$ and $I_{6}$, have little impact on the user since back-lobe antenna gains are negligible. Two major interferers, $I_{1}$ and $I_{5}$, with front- 


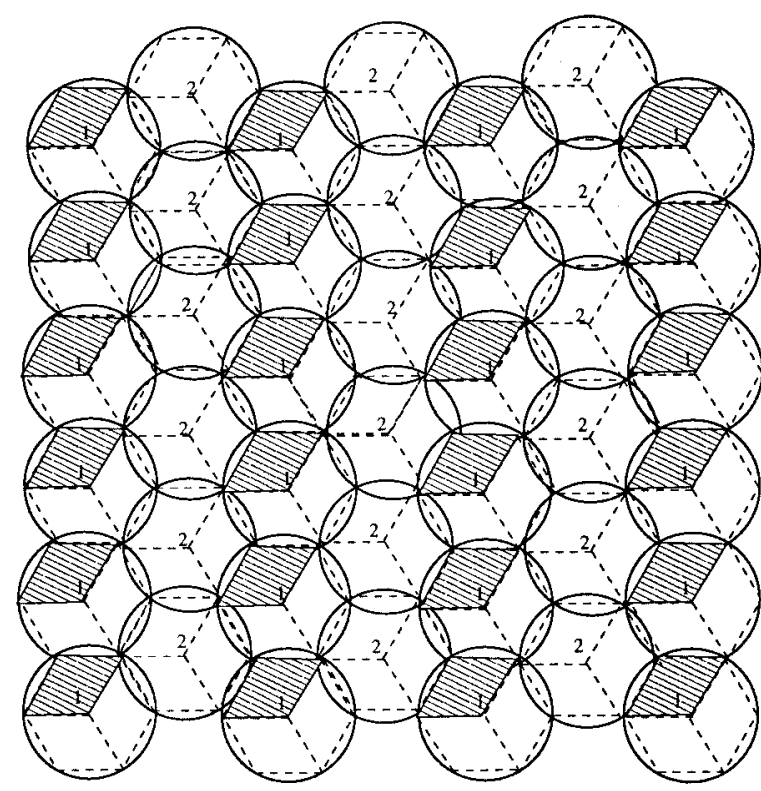

Fig. 14. An example of the WBTC system with a reuse factor $N=2$.

lobe antenna gain, are located farther away from the user. For another two interferers, $I_{3}$ has side-lobe antenna gain, and $I_{4}$ has back-lobe antenna gain. When site diversity is implemented in this scenario, a user can find four neighboring base stations that do not interfere with each other. Consequently, one can expect that, as the reuse factor becomes smaller, the performance degradation in the INBTC system is smaller than that in the WBTC and NBTC systems. In the next section, we demonstrate this property through simulation.

\section{Simulation StUdy}

\section{A. Assumptions}

We use a simulation platform, invoking the following assumptions.

1) We consider only the base-to-mobile (downlink) direction. In most cases, the downlink is the performancelimiting direction [6], [7] and therefore sufficient for study purposes.

2) In conformity with current practice in frequency-division multiple-access (FDMA) and time-division multipleaccess (TDMA) systems, we do not consider downlink power control.

3) The shadow fading components $\left(\omega_{i}, i=0,1, \cdots, n\right)$ are assumed to be mutually independent in different locations. In reality, this may not always be true, since local shadowing for a given user location can affect its paths to all base stations. Some studies have addressed this issue of correlated lognormal fading [8], but the present study does not. The shadowing loss is assumed to be constant throughout the call.

4) We consider at least two tiers of cochannel interferers.

5) We evaluate a fully loaded condition where assigned channels are constantly used, thus yielding pessimistic results. The effects of partial loading can be estimated

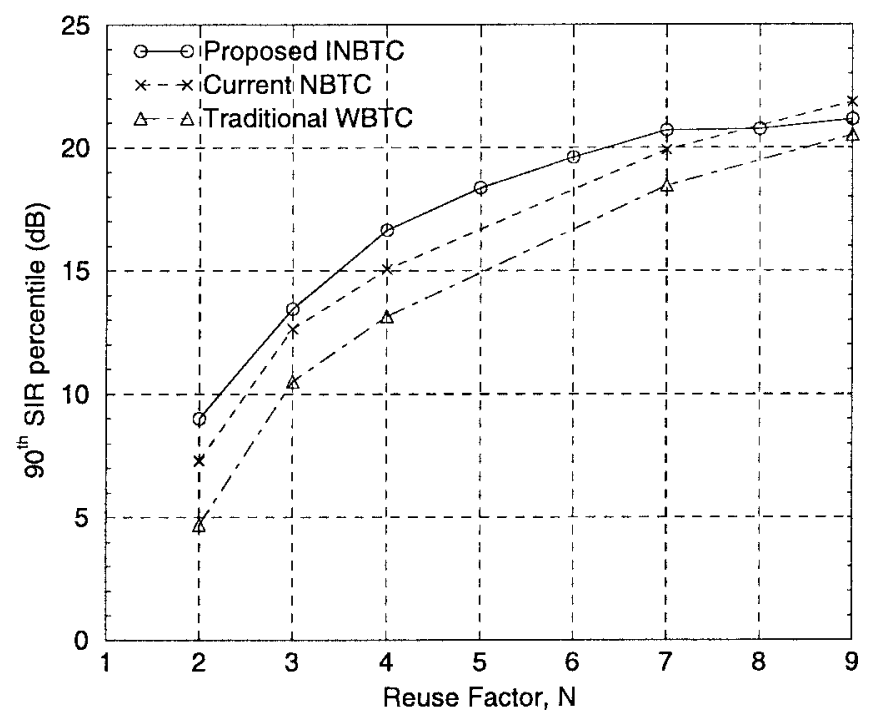

Fig. 15. Reuse efficiency comparison of the INBTC, NBTC, and WBTC systems in terms of the 90th SIR percentile. Conditions: signal strength measurement-based site diversity, downlink, fully loaded system, $\sigma=8 \mathrm{~dB}$, and $\gamma=4$.

by the method used in [4], but are not discussed in this paper.

Built upon the above assumptions, our simulation platform has been used to conduct thousands of trials via the following approach.

1) In each trial, the user population is generated randomly in a rectangular coverage area with the cell site layouts of the INBTC, NBTC, and WBTC systems (see Figs. 4-6).

2) A cell-wrapping technique is used to avoid edge effects.

3) Two kinds of macrodiversity techniques are adopted to select the serving cell: signal-based cell selection, and SIR-based cell selection.

4) The population of SIR-values so obtained has been then used to compute the reliability $P\left(\lambda_{\mathrm{th}}\right)$ [see (3)].

\section{B. Signal-Based Site Diversity}

For signal-based site diversity, cell site selection is determined by comparing measurements of signal strength from surrounding base stations. With the shadowing standard deviation $\sigma=8 \mathrm{~dB}$ and the path-loss exponent $\gamma=4$, Fig. 15 compares the reuse efficiencies of the INBTC, NBTC, and WBTC systems in terms of the 90th SIR percentile, while Fig. 16 shows the 95th SIR percentiles for the three systems. From the figures, we observe that the INBTC system provides better tradeoffs between SIR performance and reuse efficiency than the NBTC and WBTC systems, which can be elaborated upon from two different perspectives. First, the INBTC system improves the performance of SIR (or reliability) over the NBTC and WBTC systems for a given reuse factor.

- For reuse factors $N=3-7$, the INBTC system improves 3-4 dB at the 90th and 95th SIR percentiles compared with the WBTC system, and improves 1-2 dB compared with the NBTC system. 


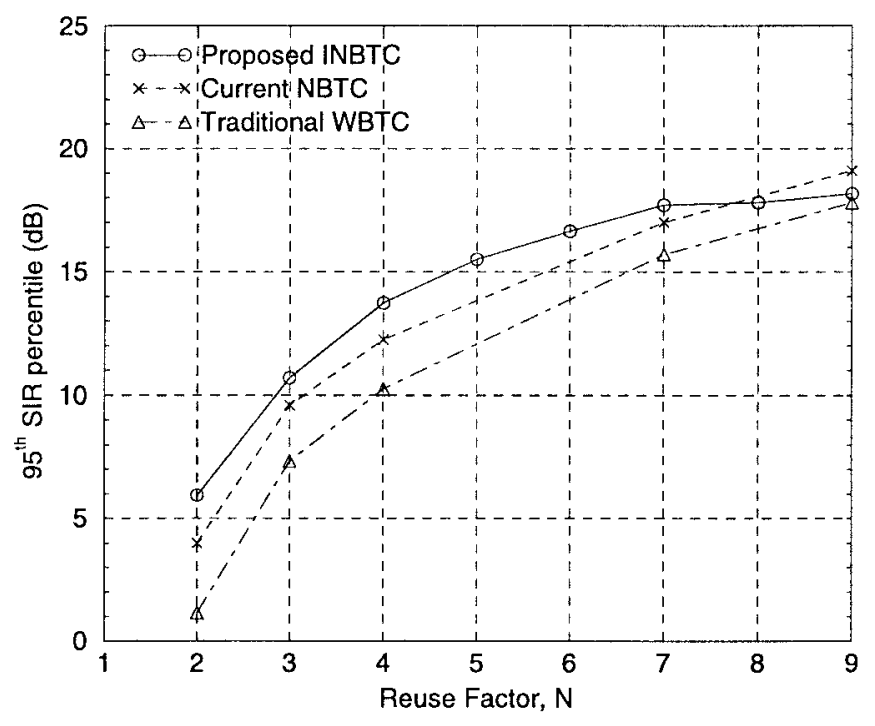

Fig. 16. Reuse efficiency comparison of the INBTC, NBTC, and WBTC systems in terms of the 95th SIR percentile. Conditions: signal strength measurement-based site diversity, downlink, fully loaded system, $\sigma=8 \mathrm{~dB}$, and $\gamma=4$.

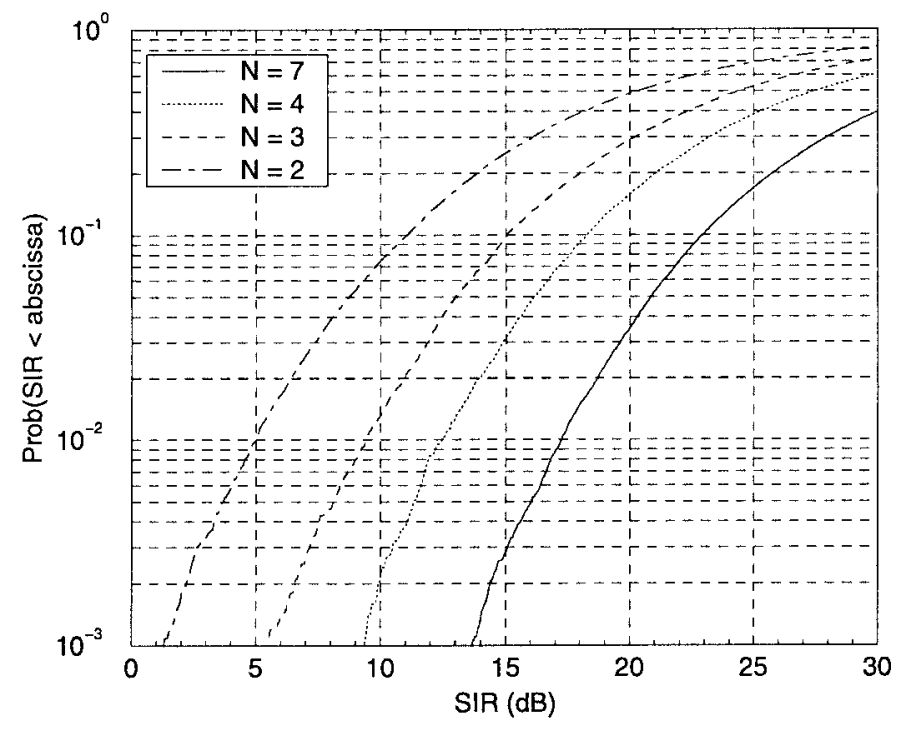

Fig. 17. SIR performance of the INBTC system with SIR measurement-based site selection diversity. Conditions: $N=2-7$, downlink, fully loaded system, $\sigma=8 \mathrm{~dB}$, and $\gamma=4$.

- For reuse factors $N=3-7$, the 95th SIR percentiles of the INBTC system are close to the 90th SIR percentiles of the WBTC system. Therefore, for the same reuse efficiency and SIR requirement, the INBTC system improves the reliability to $95 \%$ (equivalently, the outage probability is $5 \%$, or half that of the corresponding WBTC system).

Second, the INBTC system improves the capacity over the WBTC and NBTC systems for a given SIR requirement.

- The INBTC system can employ a reuse factor $N=4$ and achieve $17 \mathrm{~dB}$ for the 90th SIR percentile. However, the traditional NBTC and WBTC systems require at least $N=7$ to achieve the same performance. As a result, in this particular case, the INBTC system increases system capacity by $75 \%$ over the NBTC and WBTC systems.
TABLE V

SIR PERFormanCE OF THE INBTC SySTEM WITH SIR-Based Site Diversity in Terms of $1 \%$, 5\%, and 10\% Outage Probability

\begin{tabular}{c|c|c|c}
\hline $\mathrm{N}$ & $1 \%$ & $5 \%$ & $10 \%$ \\
\hline 2 & 5.00 & 8.75 & 11.05 \\
3 & 8.95 & 12.8 & 15.00 \\
4 & 12.45 & 16.15 & 18.3 \\
7 & 17.25 & 20.85 & 22.95 \\
\hline
\end{tabular}

- If the SIR requirement can be made lower than $10 \mathrm{~dB}$ by using advanced techniques, such as equalization, frequency hopping and interference cancellation techniques [9]-[11], the INBTC architecture can achieve both high reuse efficiency and high reliability. For example, for a 9-dB SIR requirement, as in GSM and PCS-1900 [10], the INBTC system can achieve a very high reuse efficiency $(N=2)$ with $90 \%$ reliability, while the NBTC and WBTC systems require at least $N=3$ to achieve the same performance, thus causing loss of trunking efficiency and network capacity. If the SIR requirement can be lowered to $6 \mathrm{~dB}$, then the INBTC system with $N=2$ can even reach $95 \%$ reliability.

\section{SIR-Based Site Diversity}

In the previous section, we have shown the performance improvements of the INBTC system over the NBTC and WBTC systems when using signal-based site diversity. With SIR measurement techniques, SIR-based site diversity techniques can further improve system performance. Thus, it is of interest to quantify the performance of the INBTC system with SIR-based site diversity.

Fig. 17 shows the performance of the INBTC system with SIR-based site diversity for reuse factors $N=2-7$ under the same condition used in Section V-B. Based on Fig. 17, Table $\mathrm{V}$ lists the values of SIR corresponding to outage probabilities of $1 \%, 5 \%$ and $10 \%$. Comparing the table with Figs. 15 and 16, we have the following observations.

- SIR-based site diversity improves the SIR performance of the INBTC system by $2-3 \mathrm{~dB}$ over signal-based diversity.

- The higher the percentile requirement, the higher the gain of SIR-based site diversity over signal-based site diversity. The gain is 2 and $3 \mathrm{~dB}$ at the 90th and 95th percentiles, respectively.

\section{CONCLUSION}

We have proposed a new INBTC architecture with a new ICA scheme to achieve three challenging objectives: highspectrum reuse efficiency, high-quality channels, and low infrastructure costs. From a "local" perspective, because the INBTC architecture is the same as the NBTC architecture of certain existing cellular/PCS systems, implementing the architecture will be simple and carry no extra equipment costs.

From a "global" perspective, the INBTC system, combined with the ICA scheme, can enhance performance over the conventional cellular/PCS systems in two major ways. First, it delivers higher quality channels in terms of SIR for a given 
reuse factor and coverage. For a typical range of reuse factors $N=3-7$, the INBTC system improves by $3-4 \mathrm{~dB}$ at the 90 th and 95th SIR percentiles compared to the conventional cellular systems. With a very low reuse factor $N=2$, the INBTC system achieves 9 and $11 \mathrm{~dB}$ of SIR with $90 \%$ reliability using signal-based site diversity and SIR-based site diversity, respectively.

Second, the INBTC system achieves higher frequency reuse efficiency, thus significantly improving system capacity. For a typical cellular environment with $\gamma=4, \sigma=8 \mathrm{~dB}$, and a requirement of $17 \mathrm{~dB}$ at the 90th SIR percentile, while the conventional NBTC and WBTC systems require a reuse factor $N=7$, the INBTC system can achieve the same performance with a reuse factor $N=4$, thereby increasing system capacity by $75 \%$ over the existing cellular systems.

Furthermore, the INBTC system has more flexibility to select its reuse factor. Specifically, the reuse factor of the INBTC system can be any of the integer values $N \geq 2$ (Section III-B). By contrast, the reuse factors of the NBTC and WBTC systems usually assume only certain discrete values with $N=i^{2}+i j+j^{2}$ for nonnegative integers $i$ and $j$, e.g., $N=3,4,7,9,12$, etc. Thus, the INBTC system provides additional flexibility in cell engineering without sacrificing network capacity.

A number of research topics for the INBTC system are worthwhile further investigating. First, it is desirable to quantify the impact of variations of cell locations on the INBTC system. Second, extending the interleaved cluster concept to the dynamic channel allocation systems is also an interesting research problem.

\section{ACKNOWLEDGMENT}

The author is grateful to S. Ariyavisitakul for suggesting the idea of the generalized ICA and for his help in establishing the simulation platform as well as to L. J. Greenstein and K. $\mathrm{K}$. Leung for their valuable comments and suggestions.

\section{REFERENCES}

[1] V. Palestini, "Evaluation of overall outage probability in cellular systems," in IEEE Veh. Technol. Conf., 1989, pp. 625-630.
[2] R. H. Roy, "Spatial division multiple access technology and its applications to wireless communication systems," in IEEE Veh. Technol. Conf., 1997, pp. 730-734.

[3] T. Kanai, "Channel assignment for sector cell layout," IEICE, vol. J73 B-II, no. 11, pp. 595-601, 1990.

[4] L. C. Wang, K. C. Chawla, and L. J. Greenstein, "Performance studies of narrow beam trisector cellular systems," Int. J. Wireless Inform. Networks, vol. 5, no. 2, pp. 89-102, 1998.

[5] L. C. Wang, G. L. Stüber, and C. T. Lea, "Architecture design, frequency planning, and perform analysis for a microcell/macrocell overlaying system," IEEE Trans. Veh. Technol., no. 4, pp. 836-848, 1997.

[6] M. V. Clark, V. Erceg, and L. J. Greenstein, "Reuse efficiency in urban microcellular networks," in IEEE Veh. Technol. Conf., 1996, pp. $421-425$.

[7] R. Rudokas and T. Benz, "Is it time to rethink frequency reuse formulas?," Telephony, vol. 232, pp. 68-74, Mar. 1997.

[8] S. Ariyavisitakul, T. E. Darcie, L. J. Greenstein, M. P. Phillips, and N. K. Shankaranarayanan, "Performance of simulcast wireless techniques for personal communication systems," IEEE J. Select. Areas Commun., vol. 14, pp. 632-643, May 1996.

[9] S. Ariyavisitakul and Y. Li, "Joint coding and decision feedback equalization for broadband wireless channels," in IEEE Veh. Technol. Conf., 1998, pp. 2256-2261.

[10] K. Raith and J. Uddenfeldt, "Capacity of digital cellular TDMA systems," IEEE Trans. Veh. Technol., vol. 40, pp. 323-332, May 1991.

[11] Y. Li, J. H. Winters, and N. R. Sollenberger, "Spatial-temporal equalization for IS-136 TDMA systems with rapid dispersive fading and co-channel interference," IEEE Trans. Veh. Technol., to be published.

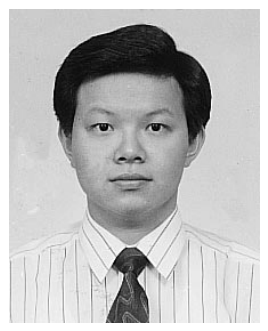

Li-Chun Wang (S'93-M'96) received the B.S. degree from National Chiao Tung University, Taiwan, R.O.C., in 1986, the M.S. degree from National Taiwan University, Taiwan, in 1988, and the M.Sci and Ph.D. degrees from the Georgia Institute of Technology, Atlanta, in 1995 and 1996, respectively, all in electrical engineering.

From 1990 to 1992, he was with the Telecommunications Laboratories of Chunghwa Telecom Corporation, Taiwan. In 1995, he was with Bell Northern Research of Northern Telecom, Inc., Richardson, TX. Since July 1996, he has been with AT\&T Laboratories, where he is a Senior Technical Staff Member in the Wireless Communications Research Department. His current research interests are in the areas of cellular architectures, radio resource management, and propagation channel modeling. Specific topics include hierarchical cellular architectures, macrodiversity cellular systems, dynamic channel allocations, power control, and microcellular interference modeling.

Dr. Wang received the 1997 IEEE Jack Neubauer Award for the best paper of the year published by the IEEE Vehicular Technology Society on the subject of vehicular technology systems. 